\title{
Controversies in the diagnosis and management of testosterone deficiency syndrome
}

\author{
Geoffrey I. Hackett MD
}

See also page 1369 and www.cmaj.ca/lookup/doi/10.1503/cmaj.150033

Competing interests: None declared.

This article was solicited and has not been peer reviewed.

Correspondence to: Geoffrey Hackett, geoff.hackett@virgin.net

CMAJ 2015. DOI:10.1503 /cmaj.151208
$\mathrm{T}$ estosterone deficiency syndrome is an area fraught with disagreement and controversy. A new Canadian guideline ${ }^{1}$ from the Canadian Men's Health Foundation is welcomed in the light of the huge volume of research on this topic over the last five years. Given the ongoing controversy and changes in our understanding of testosterone deficiency, it is not surprising that there are multiple guidelines available from other developers, including recent updates from the European Association of Urology ${ }^{2}$ and the International Society for Sexual Medicine. ${ }^{3}$

The guideline from the Canadian Men's Health Foundation ${ }^{1}$ provides recommendations specifically for Canadian physicians and is largely consistent with the 2008 International Society for the Study of the Aging Male ${ }^{4}$ and 2010 Endocrine Society guidelines. ${ }^{5}$ The current guideline will be a useful resource for Canadian physicians, but although research studies are bringing clarity to some aspects of caring for patients with testosterone deficiency syndrome, there are still many for which there is no consensus.

The diagnosis of testosterone deficiency syndrome is not straightforward. With the known limitations of testosterone measurement and lack of a valid symptom score, it is not surprising that primary care physicians lack confidence in diagnosing the syndrome, especially when experts cannot agree on values. True to the Endocrine Society guideline, ${ }^{5}$ the new Canadian guideline refers to unequivocal testosterone deficiency syndrome and

equivocal testosterone deficiency syndrome without clarifying laboratory values. ${ }^{1}$ Instead, the authors put weight on a combination of factors clinical history, physical examination and response to therapy - in making the diagnosis, in addition to measuring testosterone. The European Association of Urology and the International Society for Sexual Medicine set parameters that men with a total testosterone level of less than $8 \mathrm{nmol} / \mathrm{L}$ will usually benefit from treatment and that a trial of therapy may be indicated for those with levels between 8 and $12 \mathrm{nmol} / \mathrm{L}$ in the presence of substantial symptoms. ${ }^{2,3}$ Although experts could argue about these levels, the levels do, at least, provide a basis for primary care management and remove the mystique that only an eminent endocrinologist can really diagnose "true" testosterone deficiency. Canadian physicians may find these specific levels helpful as a guide in diagnosis.

Several guidelines recommend a trial of treatment as a component of the diagnostic process, particularly in patients with borderline testosterone levels. What is at issue is the length of the trial. The Canadian guideline advises a threemonth trial of treatment, ${ }^{1}$ as also recommended in the Endocrine Society guideline..$^{5}$ In line with the International Society for Sexual Medicine guideline, ${ }^{3}$ I suggest that clinicians consider a minimum period of six months when assessing response. In a randomized controlled trial of testosterone undecanoate in men with type 2 diabetes, my colleagues and I showed that improvement continued until six months, and even extended to 12 months in some patients. ${ }^{6}$ Contributing factors to long response times in testosterone therapy include compliance issues with topical treatment. In addition, for patients receiving testosterone undecanoate treatment, a three-month trial period would include only two long-acting injections, with peak levels not necessarily being reached. Because men are likely to get a trial of testosterone therapy only once, it is vital that we do it properly and expose them to sustained levels of testosterone for an adequate period to achieve maximal benefit. 
There is no consensus on the choice of initial biochemical testing. The Canadian guideline recommends that the total testosterone level be measured in the first instance, with determination of bioavailable testosterone using sex hormonebinding globulin only in patients with both symptoms and equivocally low total testosterone levels. ${ }^{1}$ Sex hormone-binding globulin levels may be elevated in conditions such as liver disease and hyperthyroidism, with use of certain medications such as anticonvulsants, and even in extreme age. In men with these conditions, a total testosterone level of $14 \mathrm{nmol} / \mathrm{L}$, which is within the normal range according to the European Association of Urology and International Society for Sexual Medicine guidelines, ${ }^{2,3}$ could be associated with an appropriate diagnosis of testosterone deficiency syndrome based on free or bioavailable testosterone. To identify these men may require measurement of sex hormone-binding globulins, a test that I believe is neither too complicated nor too expensive to perform just once.

It is good to see that the Canadian guideline group acknowledges erectile dysfunction as one of the most common symptoms associated with testosterone deficiency and a justification for testosterone therapy. ${ }^{1}$ Virtually all guidelines on erectile dysfunction arrive at the sensible conclusion that total testosterone level should be measured in all patients who experience erectile dysfunction. This includes men with type 2 diabetes. However, not all guidelines that focus on testosterone deficiency come to the same conclusion. The Endocrine Society guideline, for example, recommends against screening for testosterone deficiency in men with type 2 diabetes and erectile dysfunction. ${ }^{5}$ More than three-quarters of men with type 2 diabetes have erectile dysfunction, ${ }^{6}$ and about $90 \%$ have positive symptom scores for testosterone deficiency syndrome. ${ }^{6}$ Surely these facts now justify a recommendation to screen all men with type 2 diabetes for hypogonadism. The same justification for screening probably applies to men with HIV and those with chronic opioid use.

The Canadian guideline includes a caveat that, for men with erectile dysfunction and no other manifestations of testosterone deficiency syndrome, investigation should be considered only after a trial of phosphodiesterase type 5 (PDE-5) inhibitors has failed. ${ }^{1}$ It is difficult to justify this delay, because testosterone deficiency is considered a curable cause of erectile dysfunction. ${ }^{2}$ Specialists in sexual medicine realize that sexual desire, nocturnal erections, orgasm, ejaculation, intercourse and relationship satisfaction are equally important and more likely to respond to testosterone therapy than to PDE-5 inhibitors. In younger men with no comorbidities, testosterone therapy will almost cer- tainly deal with all of their symptoms. Prescribing on-demand PDE-5 inhibitors to this group of patients is unlikely to be seen as an effective solution by the couple. Also, because patients may value effective relief of symptoms over a shortterm reduction in fertility with testosterone therapy, discussion about goals of therapy is essential in making treatment decisions, a point strongly made in the Canadian guideline. ${ }^{1}$

Differing views on treatment approaches among guideline and expert groups are particularly common in the setting of comorbidities, such as obesity, diabetes and cardiac problems. In men with obesity and testosterone deficiency, the Canadian guideline rightly points out that weight loss may increase testosterone levels over two to four years. ${ }^{1}$ In a meta-analysis, Corona and colleagues ${ }^{7}$ suggested that weight reduction should be the first step for all men with obesity and testosterone deficiency. However, symptoms do not improve with weight loss alone, ${ }^{8}$ especially in men with comorbidities. ${ }^{9}$ Patients, quite rightly, demand relief of symptoms, not just improved blood levels, and hence testosterone therapy should not be delayed while awaiting possible long-term benefits from weight loss. For men with diabetes, the study by my colleagues and I showed a positive effect of testosterone treatment on glycated hemoglobin $\left(\mathrm{HbA}_{\mathrm{cc}}\right)$ concentrations. ${ }^{6}$ In addition, long-term registry studies showed sustained improvements in $\mathrm{HbA}_{1 \mathrm{c}}$ with testosterone therapy in patients with poorly controlled diabetes. ${ }^{10}$ Treatment of testosterone deficiency produces modest improvements in several modalities that, combined, may constitute considerable benefit to the patient with diabetes. ${ }^{6}$

The literature on the treatment of testosterone deficiency is rapidly changing. When therapeutic levels of testosterone therapy are achieved over a sustained period, all-cause mortality may be reduced. ${ }^{11}$ A recent meta-analysis has suggested that injection of testosterone, especially longacting formulations, has higher efficacy rates and safety benefits than topical treatment owing to the substantially lower levels of dihydrotestosterone associated with conversion of 5 - $\alpha$-reductase inhibitors in skin. ${ }^{12}$

Opinion varies on monitoring patients taking testosterone therapy. My colleagues and I showed that prostate-specific antigen (PSA) levels increased only in men treated for severe testosterone deficiency $(<8 \mathrm{nmol} / \mathrm{L}){ }^{6}$ The European Association of Urology guideline recommends that the PSA level measured six months after starting testosterone therapy be considered as the baseline against which future levels are assessed; failure to recognize this may result in excessively high biopsy rates. ${ }^{2}$ In addition to monitoring PSA and 
hematocrit at three and six months after the start of treatment and annually thereafter, the Canadian guideline suggests digital rectal examination at baseline, at six months and annually. ${ }^{1}$ Although this is graded as a weak recommendation, there is no evidence of the value of digital rectal examination in this setting, especially because it usually requires specialist consultation with associated costs. Because the evidence suggests no increased risk of prostate cancer associated with testosterone therapy, the chances of a cancer detected by digital rectal examination in a man with normal findings on examination a few months earlier and a normal PSA level must be remote.

Many will welcome the clarity provided by the new Canadian guideline and other recently revised guidelines, but many important clinical issues remain unresolved. Unfortunately, it is unlikely that the ideal clinical trials required to provide the highest levels of evidence will ever be done for ethical, practical and financial reasons.

\section{References}

1. Morales A, Bebb RA, Manjoo P, et al.; Canadian Men's Health Foundation Multidisciplinary Guidelines Task Force on Testosterone Deficiency. Diagnosis and management of testosterone deficiency syndrome in men: clinical practice guideline. CMAJ 2015;187:1369-77.

2. Dohle GR, Arver S, Bettocchi C, et al. Guidelines on male hypogonadism. Arnhem (the Netherlands): European Association of
Urology; 2014. Available: http://uroweb.org/wp-content/uploads/ 18-Male-Hypogonadism_LR.pdf (accessed 2015 Oct. 1).

3. Dean JD, McMahon C, Guay A, et al. The International Society for Sexual Medicine's process of care for the assessment and management of testosterone deficiency in adult men. $J$ Sex Med 2015;12:1660-86.

4. Lunenfeld B, Mskhalaya G, Zitzmann M, et al. Recommendations on the diagnosis, treatment and monitoring of hypogonadism in men. Aging Male 2015;18:5-15.

5. Bhasin S, Cunningham GR, Hayes FJ, et al.; Task Force, Endocrine Society. Testosterone therapy in men with androgen deficiency syndromes: an Endocrine Society clinical practice guideline. J Clin Endocrinol Metab 2010;95:2536-59.

6. Hackett G, Cole N, Bhartia M, et al.; Blast Study Group. The response to testosterone undecanoate in men with type 2 diabetes is dependent on achieving threshold serum levels (the BLAST study). Int J Clin Pract 2014;68:203-15.

7. Corona G, Rastrelli G, Maggi M. Diagnosis and treatment of late onset hypogonadism: systematic review and meta-analysis of TRT outcomes. Best Pract Res Clin Endocrinol Metab 2013;27:557-79.

8. Rastrelli G, Carter EL, Ahern T, et al.; EMAS Study Group. Development of and recovery from secondary hypogonadism in aging men: prospective results from the EMAS. J Clin Endocrinol Metab 2015;100:3172-82.

9. Wing RR, Rosen RC, Fava JL, et al. Effects of weight loss intervention on erectile function in older men with type 2 diabetes in the Look AHEAD trial. $J$ Sex Med 2010;7:156-65.

10. Saad F, Yassin A, Doros G, et al. Effects of long-term treatment with testosterone on weight and waist size in 411 hypogonadal men with obesity classes I-III: observational data from two registry studies. Int J Obes (Lond) 2015 July 29 [Epub ahead of print].

11. Sharma R, Oni OA, Gupta K, et al. Normalization of testosterone level is associated with reduced incidence of myocardial infarction and mortality in men. Eur Heart J 2015 Aug. 6 [Epub ahead of print].

12. Borst SE, Yarrow J. Injection of testosterone may be safer and more effective than transdermal administration for combating loss of muscle and bone in older men. Am J Physiol Endocrinol Metab 2015;308:E1035-42.

Affiliation: Heartlands Hospital, Birmingham, UK

\section{REPRINTS}

To purchase commercial article reprints and e-prints or to request a quote, please contact

Matt Neiderer

Content Sales Sheridan Content Services
$800635-7181 \times 8265$ matt.neiderer@sheridan.com 